\title{
Supplementary to
}

\section{Anomalous behavior of heat capacity in $\mathrm{Ni}_{2}(\text { bdc })_{2}$ (dabco). Schottky anomaly and spin-phonon interaction}

Svetlana G. Kozlova ${ }^{1.2 *}$, Denis P. Pishchur ${ }^{1}$, Nikolay B. Kompankov ${ }^{1}$, Vladimir R. Shayapov ${ }^{1}$,

Denis G. Samsonenko ${ }^{1,3}$

1) Nikolaev Institute of Inorganic Chemistry, Siberian Branch of Russian Academy of Science, Lavrenteva Av., 3, RU-630090 Novosibirsk, Russian Federation

2) Belgorod State Technological University named after V.G. Shukhov, Kostukov Av., 46, RU308012 Belgorod, Russian Federation

${ }^{3)}$ Novosibirsk State University, Pirogova Street, 2, RU-630090 Novosibirsk, Russian Federation

Table $S 1$. Experimental values molar heat capacities $C_{p}$, and calculated thermodynamic functions of (enthalpy $\Delta \mathrm{H}=\mathrm{H}^{0}(\mathrm{~T})-\mathrm{H}^{0}(0)$ and entropy $\left.\Delta \mathrm{S}=\mathrm{S}^{0}(\mathrm{~T})-\mathrm{S}^{0}(0)\right)$ for Ni-DMOF $\left(\mathrm{M}=557.79 \mathrm{~g} \cdot \mathrm{mol}^{-1}\right)$ with correction for heat capacity of helium in the measuring ampoule.

\begin{tabular}{|l|l|l|l|}
\hline $\mathrm{T} / \mathrm{K}$ & $\mathrm{C}_{\mathrm{p}} / \mathrm{J} \bullet \mathrm{mol}^{-1} \bullet \mathrm{K}^{-1}$ & $\Delta \mathrm{H} / \mathrm{J} \bullet \mathrm{mol}^{-1}$ & $\Delta \mathrm{S} / \mathrm{J} \bullet \mathrm{mol}^{-1} \cdot \mathrm{K}^{-1}$ \\
\hline 0 & 0 & 0 & 0 \\
5.38759 & 2.63248 & 4.22601 & 1.0848 \\
5.53824 & 2.74636 & 4.63116 & 1.15896 \\
6.76817 & 4.3536 & 8.99739 & 1.85949 \\
7.47354 & 5.19158 & 12.36386 & 2.33135 \\
7.87914 & 6.63224 & 14.76174 & 2.64294 \\
8.82551 & 8.88594 & 22.1047 & 3.51766 \\
9.81658 & 10.9947 & 31.95618 & 4.57159 \\
10.85502 & 13.42616 & 44.63605 & 5.79533 \\
11.98289 & 15.56546 & 60.98542 & 7.22538 \\
13.22223 & 18.46765 & 82.07477 & 8.89582 \\
14.20561 & 20.2097 & 101.09192 & 10.28206 \\
15.09047 & 21.74111 & 119.65228 & 11.54891 \\
16.48049 & 23.98849 & 151.43468 & 13.56185 \\
17.37315 & 25.59165 & 173.56378 & 14.86899 \\
18.17458 & 27.2743 & 194.74804 & 16.06061 \\
19.61565 & 30.1182 & 236.10129 & 18.24823 \\
20.46976 & 31.82841 & 262.5561 & 19.56797 \\
21.25152 & 33.35052 & 288.0331 & 20.78916 \\
22.70775 & 36.74565 & 339.07125 & 23.11005 \\
23.54535 & 38.71135 & 370.67254 & 24.4763 \\
24.31471 & 40.47097 & 401.1324 & 25.74905 \\
25.7535 & 44.70497 & 462.40773 & 28.19525 \\
26.61874 & 46.9874 & 502.07557 & 29.70988 \\
27.3575 & 49.26297 & 537.62856 & 31.02706 \\
28.79251 & 54.36717 & 611.98364 & 33.67389 \\
30.37511 & 59.33744 & 701.95784 & 36.71385 \\
31.96535 & 65.47832 & 801.20175 & 39.89585 \\
35.00656 & 76.58936 & 1017.23039 & 46.33755 \\
38.0341 & 87.84853 & 1266.15129 & 53.14585 \\
41.04829 & 98.96627 & 1547.69883 & 60.2604 \\
\hline & & & \\
\hline
\end{tabular}




\begin{tabular}{|c|c|c|c|}
\hline 44.04238 & 109.09736 & 1859.17981 & 67.57807 \\
\hline 47.03416 & 120.14834 & 2202.106 & 75.10477 \\
\hline 50.03075 & 128.96194 & 2575.34731 & 82.79425 \\
\hline 53.02497 & 139.43416 & 2977.16515 & 90.59006 \\
\hline 56.02499 & 147.88701 & 3408.15002 & 98.494 \\
\hline 59.00739 & 157.61295 & 3863.71229 & 106.41338 \\
\hline 61.97699 & 164.67942 & 4342.25123 & 114.32464 \\
\hline 64.93508 & 173.10446 & 4841.84947 & 122.19748 \\
\hline 67.8992 & 180.74035 & 5366.26732 & 130.09344 \\
\hline 70.86287 & 187.87795 & 5912.50038 & 137.96672 \\
\hline 73.80642 & 195.50319 & 6476.75044 & 145.76734 \\
\hline 76.76004 & 204.05717 & 7066.82602 & 153.60514 \\
\hline 79.72707 & 211.29546 & 7683.00745 & 161.48054 \\
\hline 84.95026 & 225.27846 & 8823.16066 & 175.32752 \\
\hline 89.88609 & 238.6589 & 9968.11938 & 188.42478 \\
\hline 94.85996 & 247.78565 & 11177.87428 & 201.52407 \\
\hline 100.97044 & 261.5374 & 12733.9797 & 217.41854 \\
\hline 104.90942 & 268.27543 & 13777.44087 & 227.55638 \\
\hline 110.32031 & 281.02117 & 15263.53098 & 241.3664 \\
\hline 113.10297 & 287.15918 & 16054.05828 & 248.44304 \\
\hline 115.7192 & 292.14582 & 16811.85567 & 255.06672 \\
\hline 120.32938 & 302.4014 & 18182.34145 & 266.67913 \\
\hline 124.97653 & 308.8221 & 19602.56581 & 278.26019 \\
\hline 127.65537 & 312.64197 & 20434.96516 & 284.85032 \\
\hline 130.33618 & 320.37015 & 21283.45771 & 291.42788 \\
\hline 134.97078 & 329.11058 & 22788.50191 & 302.77435 \\
\hline 139.57279 & 338.89221 & 24325.57753 & 313.97207 \\
\hline 144.98427 & 349.09628 & 26187.09484 & 327.05672 \\
\hline 149.72321 & 357.40643 & 27861.13227 & 338.41818 \\
\hline 154.2926 & 367.24374 & 29516.73616 & 349.30999 \\
\hline 159.19373 & 376.58433 & 31339.53715 & 360.93977 \\
\hline 164.0179 & 388.98361 & 33186.15128 & 372.36621 \\
\hline 168.88055 & 398.317 & 35100.33458 & 383.86677 \\
\hline 173.7416 & 407.59664 & 37059.12711 & 395.30133 \\
\hline 178.60355 & 416.3897 & 39062.218 & 406.67187 \\
\hline 183.44364 & 424.13855 & 41096.33662 & 417.90926 \\
\hline 188.2676 & 432.65768 & 43162.90997 & 429.02894 \\
\hline 193.10242 & 441.31232 & 45275.65383 & 440.10908 \\
\hline 197.94756 & 450.26707 & 47435.56809 & 451.15615 \\
\hline 203.07298 & 460.34842 & 49769.2132 & 462.79493 \\
\hline 207.91349 & 469.88682 & 52020.61905 & 473.75124 \\
\hline 212.71446 & 478.76411 & 54297.83778 & 484.5792 \\
\hline 217.54131 & 485.95289 & 56626.11425 & 495.40241 \\
\hline 222.39491 & 495.83851 & 59008.72415 & 506.23414 \\
\hline 227.20975 & 504.32457 & 61416.53514 & 516.94519 \\
\hline 232.04978 & 514.09386 & 63881.12618 & 527.67819 \\
\hline 236.91877 & 522.84439 & 66405.545 & 538.44424 \\
\hline 241.75268 & 531.11722 & 68952.92442 & 549.08802 \\
\hline 246.6139 & 540.39331 & 71557.34703 & 559.75401 \\
\hline 251.50586 & 549.50613 & 74223.22145 & 570.45791 \\
\hline 256.36264 & 557.7904 & 76912.16779 & 581.04727 \\
\hline 261.17777 & 567.75877 & 79621.99834 & 591.51927 \\
\hline
\end{tabular}




\begin{tabular}{|l|l|l|l|}
\hline 266.01413 & 576.62924 & 82389.33398 & 602.0178 \\
270.88656 & 585.70205 & 85221.0267 & 612.56622 \\
275.72955 & 593.63028 & 88076.77459 & 623.01524 \\
280.54299 & 601.79961 & 90953.83633 & 633.35948 \\
285.3901 & 611.64486 & 93894.68524 & 643.75244 \\
290.27426 & 621.25321 & 96905.52089 & 654.21288 \\
295.12541 & 632.63036 & 99946.91084 & 664.60363 \\
\hline
\end{tabular}

Table S2. Smoothed molar thermodynamic functions (heat capacity $C_{p}$, enthalpy $\Delta H=H^{0}(T)-$ $\mathrm{H}^{0}(0)$ and entropy $\left.\Delta \mathrm{S}=\mathrm{S}^{0}(\mathrm{~T})-\mathrm{S}^{0}(0)\right)$ for Ni-DMOF $\left(\mathrm{M}=557.79 \mathrm{~g} \cdot \mathrm{mol}^{-1}\right)$ with a correction for heat capacity of helium in the measuring ampoule.

\begin{tabular}{|c|c|c|c|}
\hline $\mathrm{T} / \mathrm{K}$ & $\mathrm{C}_{\mathrm{p}} / \mathrm{J} \cdot \mathrm{mol}^{-1} \cdot \mathrm{K}^{-1}$ & $\Delta \mathrm{H} / \mathrm{J} \bullet \mathrm{mol}^{-1}$ & $\Delta \mathrm{S} / \mathrm{J} \cdot \mathrm{mol}^{-1} \cdot \mathrm{K}^{-1}$ \\
\hline 0 & 0 & 0 & 0 \\
\hline 5 & 2.2491 & 3.28321 & 0.91048 \\
\hline 6 & 3.31983 & 6.06767 & 1.41204 \\
\hline 7 & 4.62794 & 10.04156 & 2.01926 \\
\hline 8 & 6.93336 & 15.82221 & 2.78316 \\
\hline 9 & 9.25988 & 23.91883 & 3.73093 \\
\hline 10 & 11.4111 & 34.25432 & 4.81593 \\
\hline 11 & 13.73106 & 46.8254 & 6.01062 \\
\hline 12 & 15.60148 & 61.49167 & 7.28482 \\
\hline 13 & 18.01724 & 78.30103 & 8.62785 \\
\hline 14 & 19.85147 & 97.23538 & 10.0298 \\
\hline 15 & 21.58613 & 117.95418 & 11.45832 \\
\hline 16 & 23.21166 & 140.35308 & 12.90323 \\
\hline 17 & 24.88715 & 164.40249 & 14.36057 \\
\hline 18 & 26.91633 & 190.30423 & 15.84022 \\
\hline 19 & 28.90084 & 218.21281 & 17.34844 \\
\hline 20 & 30.88714 & 248.1068 & 18.88117 \\
\hline 21 & 32.82882 & 279.96478 & 20.43498 \\
\hline 22 & 35.07828 & 313.91834 & 22.01386 \\
\hline 23 & 37.43134 & 350.17315 & 23.62481 \\
\hline 24 & 39.74383 & 388.76073 & 25.26654 \\
\hline 25 & 42.41567 & 429.84048 & 26.94285 \\
\hline 26 & 45.36202 & 473.72933 & 28.66351 \\
\hline 27 & 48.11431 & 520.4675 & 30.42686 \\
\hline 28 & 51.52931 & 570.28931 & 32.23803 \\
\hline 29 & 55.04671 & 623.57732 & 34.10728 \\
\hline 30 & 58.07141 & 680.13638 & 36.02422 \\
\hline 35 & 76.56502 & 1016.72747 & 46.33243 \\
\hline 40 & 95.11782 & 1445.93457 & 57.74622 \\
\hline 45 & 112.59303 & 1965.2117 & 69.94626 \\
\hline 50 & 128.86284 & 2568.85136 & 82.64457 \\
\hline 55 & 144.98275 & 3253.46532 & 95.67783 \\
\hline 60 & 160.0951 & 4016.15993 & 108.93859 \\
\hline 65 & 173.27629 & 4849.58841 & 122.27369 \\
\hline 70 & 185.78384 & 5747.23874 & 135.5733 \\
\hline 75 & 198.94116 & 6709.05123 & 148.83981 \\
\hline 80 & 212.022 & 7736.45913 & 162.09687 \\
\hline 85 & 225.41205 & 8830.04426 & 175.35232 \\
\hline 90 & 238.95536 & 9990.96277 & 188.61974 \\
\hline
\end{tabular}




\begin{tabular}{|l|l|l|l|}
\hline 95 & 248.0803 & 11208.55191 & 201.78581 \\
100 & 259.47814 & 12477.448 & 214.8012 \\
105 & 268.47668 & 13797.33503 & 227.68045 \\
110 & 280.29315 & 15169.25962 & 240.44305 \\
115 & 290.77263 & 16596.92409 & 253.1345 \\
120 & 301.87369 & 18078.5399 & 265.74468 \\
125 & 308.8549 & 19605.36137 & 278.21081 \\
130 & 319.56974 & 21176.42296 & 290.53348 \\
135 & 329.17137 & 22798.27572 & 302.77482 \\
140 & 339.7302 & 24470.52964 & 314.93719 \\
145 & 349.12515 & 26192.66801 & 327.0232 \\
150 & 357.94022 & 27960.33144 & 339.00827 \\
155 & 368.65587 & 29776.82167 & 350.92 \\
160 & 378.45919 & 31644.60932 & 362.77949 \\
165 & 390.87638 & 33567.94824 & 374.61529 \\
170 & 400.45589 & 35546.27891 & 386.42671 \\
175 & 409.95759 & 37572.3126 & 398.17231 \\
180 & 418.6742 & 39643.89207 & 409.84376 \\
185 & 426.86879 & 41757.74954 & 421.42718 \\
190 & 435.75042 & 43914.29755 & 432.92924 \\
195 & 444.73493 & 46115.51091 & 444.36452 \\
200 & 454.29808 & 48363.09343 & 455.74498 \\
205 & 464.14496 & 50659.20102 & 467.08401 \\
210 & 473.85862 & 53004.20996 & 478.38549 \\
215 & 482.17758 & 55394.30046 & 489.63338 \\
220 & 490.93374 & 57827.07875 & 500.81889 \\
225 & 500.38623 & 60305.37867 & 511.95753 \\
230 & 510.0122 & 62831.37474 & 523.06098 \\
235 & 519.45341 & 65405.03876 & 534.13069 \\
240 & 528.04236 & 68023.77818 & 545.15724 \\
245 & 537.3075 & 70687.15283 & 556.14041 \\
250 & 546.71999 & 73397.22157 & 567.09034 \\
255 & 555.40184 & 76152.52615 & 578.00265 \\
260 & 565.42028 & 78954.58145 & 588.8845 \\
265 & 574.76297 & 81805.03959 & 599.74353 \\
270 & 584.09177 & 84702.17643 & 610.57408 \\
275 & 592.43756 & 87643.49976 & 621.36813 \\
280 & 600.8069 & 90626.61093 & 632.11827 \\
285 & 610.85743 & 93655.77175 & 642.84102 \\
290 & 620.66784 & 96734.58491 & 653.55 \\
295 & 632.31323 & 99867.03756 & 664.25917 \\
295.12541 & 632.63036 & 99946.35585 & 664.52799 \\
298.15 & 640.64867 & 101871.92936 & 671.01928 \\
\hline & & & \\
\hline
\end{tabular}




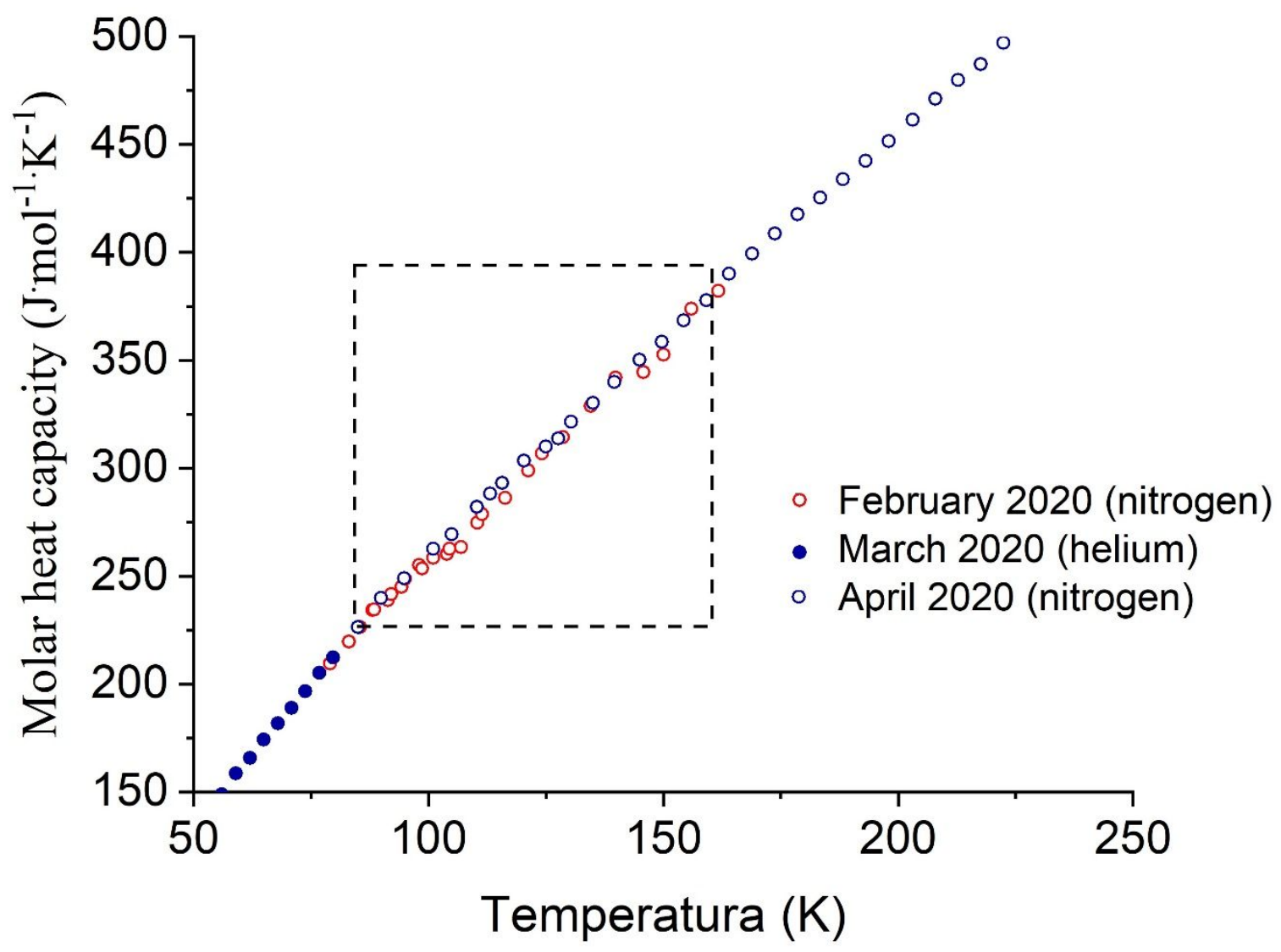

Figure S1. Scattering of experimental molar heat capacity $\left(C_{p}\right)$ values in the region of $80-160$ $\mathrm{K}$ in Ni-DMOF. The inset shows the time when the experiments were conducted and the cooling agent. 


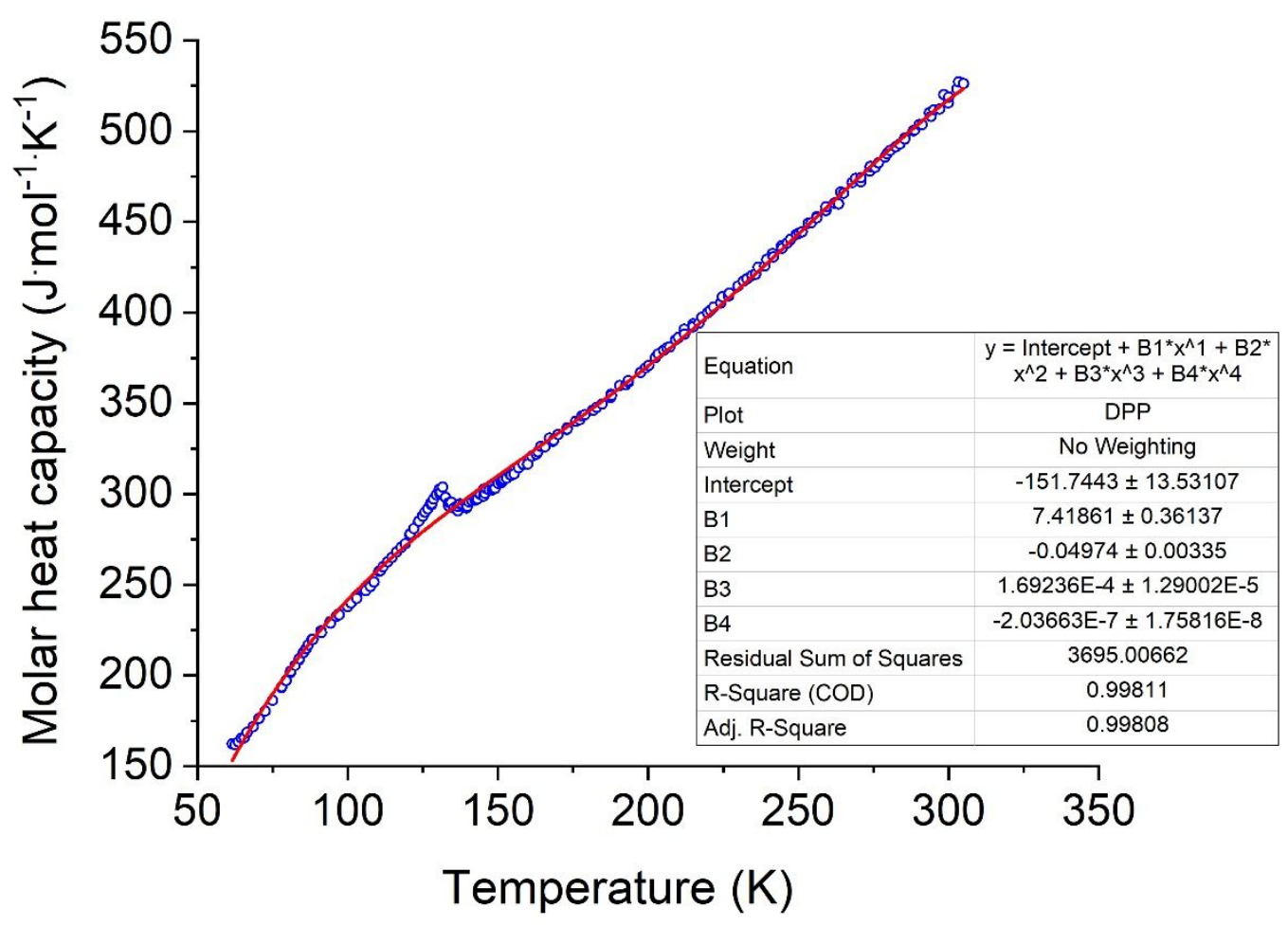

Figure S2. Modeling the molar heat capacity $\left(\mathrm{C}_{\mathrm{p}}\right)$ of $\mathrm{Zn}$-DMOF by a polynomial of degree 4 . 